\title{
GeriMedRisk, a telemedicine geriatric pharmacology consultation service to address adverse drug events in long-term care: a stepped-wedge cluster randomized feasibility trial protocol (ISRCTN17219647)
}

\author{
Joanne Man-Wai Ho ${ }^{1,2,3^{*}}$ (D) Jennifer Tung ${ }^{3}$, Janine Maitland ${ }^{4}$, Derelie Mangin ${ }^{5}$, Lehana Thabane ${ }^{6}$, J. Michael Pavlin',
} Jeffrey Alfonsi ${ }^{8}$, Anne Holbrook ${ }^{6,9}$, Sharon Straus ${ }^{10,11}$ and Sophiya Benjamin ${ }^{1,3}$

\begin{abstract}
Background: Multimorbidity, polypharmacy, and older age predispose seniors to adverse drug events (ADE). Seniors with an ADE experience greater morbidity, mortality, and health care utilization compared to their younger counterparts. To mitigate and manage ADEs among this vulnerable population, we designed a geriatric pharmacology consultation service connecting clinicians with specialist physicians and pharmacists and will investigate the feasibility and acceptability of this complex intervention in the long-term care setting, prior to conducting a larger efficacy trial.

Methods/Design: We will conduct a cluster randomized feasibility trial and qualitative analysis of GeriMedRisk among four long-term care homes in the Waterloo-Wellington region from May 1 to December 31, 2017. The primary outcome is the feasibility and acceptability of GeriMedRisk and the stepped-wedge cluster randomized controlled trial design. We hypothesize that GeriMedRisk is a feasible intervention and its potential to decrease falls and drug-related hospital visits can be evaluated with a stepped-wedge cluster randomized controlled trial design.
\end{abstract}

Discussion: This mixed methods study will inform a larger efficacy trial of GeriMedRisk's ability to decrease adverse drug events among seniors in the long-term care setting.

Ethics and dissemination: The Hamilton Integrated Research Ethics Board granted the approval for this study protocol 2812. We plan to disseminate the results of this study in peer-reviewed journals and also to our partners and stakeholders.

Trial registration: ISRCTN clinical trials registry, ISRCTN17219647 (March 27, 2017)

Keywords: Geriatrics, Appropriate prescribing, Telemedicine, Clinical trial, Feasibility, Cluster randomized controlled trial

\section{Background}

Every year, tens of thousands of seniors experience a poisoning or adverse drug event (ADE) [1, 2]. Multiple diseases, polypharmacy, and age predispose older adults to drug toxicity. Following a poisoning, seniors (aged $\geq 65$ years) are four times more likely to die or to require hospitalization compared to their younger

\footnotetext{
* Correspondence: joanneho@mcmaster.ca

${ }^{1}$ Waterloo Regional Campus, McMaster University DeGroote School of Medicine, $10 B$ Victoria St S, Kitchener, ON, Canada

${ }^{2}$ Schlegel Research Institute for Aging, 250 Laurelwood Drive, Waterloo, ON, Canada Full list of author information is available at the end of the article
}

counterparts [3]. Drug toxicity is costly, with at least $\$ 13$ million spent on ADE-related hospital visits among seniors and $\$ 419$ million spent annually on potentially inappropriate medications in North America [1, 4]. Decreasing polypharmacy may prevent ADEs among seniors. Studies of deprescribing interventions among older adults found improved quality of life and decreased hospitalization and mortality [5]. Widespread application is limited, however, by inadequate systems of care integrating primary care, pharmacy, geriatric medicine, and clinical pharmacology [6, 7]. Pharmacist-led medication review services to assess medication appropriateness

(c) The Author(s). 2018 Open Access This article is distributed under the terms of the Creative Commons Attribution 4.0 International License (http://creativecommons.org/licenses/by/4.0/), which permits unrestricted use, distribution, and 
include the Medscheck and the Pharmaceutical Opinion Programs. The Ontario Pharmacy Research Network (OPEN), however, found that a minority of patients receiving a Medscheck were older and medically complex and that there was a discrepancy in the quality of the delivered service, and there was no evidence of improved patient outcomes [8]. Furthermore, existing deprescribing algorithms and apps may not apply to patients with multimorbidity or mental illness, a group vulnerable to ADEs [9]. The majority of expertise in geriatric pharmacology is concentrated in urban academic health sciences centers resulting in inequitable access for Canadians residing in rural and remote areas [10]. There is a need for a timely and cost-effective geriatric pharmacology consultation service for Ontario seniors irrespective of their location.

Telemedicine, or telehealth, is the use of communication technologies to deliver patient care and medical services remotely [11]. It enables the delivery of health care services to areas that are remote or that have insufficient clinicians and resources [11]. Telemedicine tools include telephone and computer- or mobile device-based videoconference or eConsult $[11,12]$. Its use improves access to care, support from specialists to primary care, and potentially decreases health care costs [11, 13-15]. Telemedicine services have been able to increase access to specialist clinician services for toxicology through poison centers, stroke, psychiatry, dermatology, and hepatic disease [11, 16-19]. With the limited number of clinicians with specialization in geriatrics, telemedicine may be a potential solution to serve the increasing aging population $[10,14,15]$.

GeriMedRisk is a novel interdisciplinary, technology-based geriatric pharmacology consultation service that aims to optimize a patient's medications to improve cognition, mobility, function, and mental health by supporting their clinicians. Referring clinicians will be able to easily access GeriMedRisk nurses, pharmacists, and physicians specializing in geriatric medicine, clinical pharmacology, and geriatric psychiatry by telephone or through telemedicine. By supporting clinicians as they optimize their complex older patients' medications, GeriMedRisk has the potential to decrease drug-related cognitive impairment, falls, and hospital visits among seniors from all clinical settings. Prior to conducting an efficacy trial of GeriMedRisk to decrease adverse drug events across 14 long-term care (LTC) homes in Southwestern Ontario, we will first test the feasibility of the service and proposed study design, a stepped-wedge cluster randomized controlled trial.

The aim of this study is to investigate the feasibility and acceptability of the GeriMedRisk and its evaluation with a stepped-wedge randomized controlled trial in the long-term care (LTC) setting. We also intend to identify indicators of a difference in falls and drug-related hospital visits among seniors residing in LTC with access to GeriMedRisk compared to those without. We hypothesize that GeriMedRisk is a feasible intervention, and its potential to decrease falls and drug-related hospital visits can be evaluated with a stepped-wedge cluster randomized controlled trial design.

\section{Methods}

This protocol (version 4.0, March 27, 2017) was developed in concordance with the Standardized Protocol Item: Recommendations for Interventional Trials (SPIRIT) checklist and CONSORT extension [20] to pilot trials and is registered with the ISRCTN clinical trials registry (ISRCTN17219647). The trial sponsor is McMaster University located at 1280 Main St. W., Hamilton, Ontario, L8S 4L8, Canada.

\section{Setting}

We will conduct this study across a convenience sample of four LTC facilities in the Waterloo-Wellington region, Ontario, Canada, with each site serving as a cluster. The Waterloo-Wellington region has a population exceeding 775,000 residents and encompasses the major urban centers of Waterloo, Kitchener, Cambridge, and Guelph. While $90 \%$ of the geography is rural, $90 \%$ of the population lives in urban areas. The population of Waterloo-Wellington has similar demographics as the rest of the province of Ontario [21]. At each site, residents are assigned to a primary care doctor or nurse practitioner. Each site is supported by a consultant pharmacist who performs medication reviews at full-time equivalents ranging between 0.25 and 0.3 . The three Schlegel Village LTC homes have uniform physical environments and philosophies of care but individualized staffing and clinical programs. University Gates Schlegel Village is a 192-bed LTC home located in Waterloo, supported by primary care clinicians and regular on-site geriatric medicine and geriatric psychiatry consultants. Winston Park Schlegel Village located in Kitchener is a 95-bed facility supported by a primary care clinician and an on-site geriatrician consultant. Riverside Glen Schlegel Village is a 192-bed LTC home located in Guelph that does not have designated support from a geriatrician. LTC patients that require a geriatrician are referred through a centralized intake process through the Waterloo Wellington Specialized Geriatrics Services. St. Joseph's Health Centre Guelph, a non-profit 244-bed facility, is supported by primary care physician and an on-site consultant geriatrician.

\section{Eligibility criteria}

Inclusion criteria: 
- Cluster: a convenience sample of LTC homes in Waterloo-Wellington region that expressed interest in participating in this feasibility study.

- Individual participants: all physicians, pharmacists, and nurse practitioners who provide patient care in a participating LTC site when they are allocated to the intervention.

Exclusion criteria:

- There are no exclusion criteria for LTC clinicians or residents.

Criteria for discontinuing the intervention:

- If the referring clinician's patient status is an acute poisoning (defined as an ingestion of more than two times the prescribed dose), or clinically unstable, GeriMedRisk will abort the call and encourage the clinician to call either the Ontario Poison Centre and/or emergency services.

\section{Intervention}

In addition to the usual Waterloo-Wellington regional geriatric services, clinicians caring for seniors with medication challenges will have access to GeriMedRisk. GeriMedRisk is an interdisciplinary, technology-based geriatric pharmacology consultation and review service. It aims to optimize a patient's medications to improve cognition, mobility, function, and mental health by supporting their clinicians. Referring clinicians will be able to easily access GeriMedRisk nurses, pharmacists, and physicians specializing in geriatric medicine, clinical pharmacology, and geriatric psychiatry by telephone or through telemedicine during business hours. Input from the following informed GeriMedRisk's development: long-term care residents and their caregivers; Waterloo-Wellington region health administrators; the Ontario Poison Centre, a provincial service that uses telemedicine to deliver toxicological expertise to the public and clinicians in Ontario, Canada; the Ontario Telemedicine Network; and Champlain BASE eConsult and a needs assessment of Waterloo-Wellington physicians, nurse practitioners, pharmacists about geriatric pharmacotherapy.

Participants have two options for methods for referral:

1. Telephone: this traditional mode of person-to-person consultation can be either real-time or delayed by a few hours if used as a paging service whereby the referring clinician leaves a call-back number.

2. eConsult: the referring clinician sends an eConsult with the patient's necessary clinical information through a private and secure portal through the
Ontario Telemedicine Network (OTN) at www.otnhub.ca to GeriMedRisk [12]. The GeriMedRisk team responds within five business days through eConsult with a request for additional information or recommendations. Additional support through the phone, OTN videoconference or in-person may be arranged if necessary.

The GeriMedRisk staff will collaborate with the referring clinician to perform a geriatric pharmacology consultation and medication review or answer a geriatric drug information question. The former process utilizes components from a comprehensive geriatric assessment to address the patient's cognition, comorbidities, mobility, function, and mental health and incorporates an intrinsic medication appropriateness assessment [22, 23]. Videoconference telemedicine and in-person consultations with specialist physicians across Southwestern Ontario academic health centers (McMaster University, University of Toronto, University of Western Ontario) would be available if necessary. Password-protected consultation reports and user-friendly drug information and knowledge translation materials will be securely sent to the referring clinician through OTN eConsult. These educational materials are concise evidence-based documents relevant to the consult that serve to build geriatric pharmacotherapy capacity among referring clinicians. Their development is in accordance with rapid review methodology described by the National Collaborating Centre for Methods and Tools [24]. GeriMedRisk will perform at least one follow-up to the referring clinician within 2 weeks to assess the effectiveness of the recommendations and monitor adherence. During the duration of the study period, all LTC sites would continue to have access to their usual local and regional geriatric services. There will be no restrictions in concomitant care and interventions. The control period is defined as the 12-month period preceding the day the LTC's clinicians acquired access GeriMedRisk.

\section{Study design}

We will conduct a mixed methods feasibility study using an exploratory framework. The quantitative component will be a stepped-wedge cluster randomized controlled trial. All study sites will have uniform access to their usual Waterloo Wellington Local Health Integration Network geriatric services. Using a computer-generated sequence and simple randomization techniques, we will randomize the order of when each site acquires access to GeriMedRisk. Every 8 weeks, a new site will gain access to the intervention until all four LTC sites have access to the intervention (Fig. 1). This time period allows for the orientation and potential adoption of 


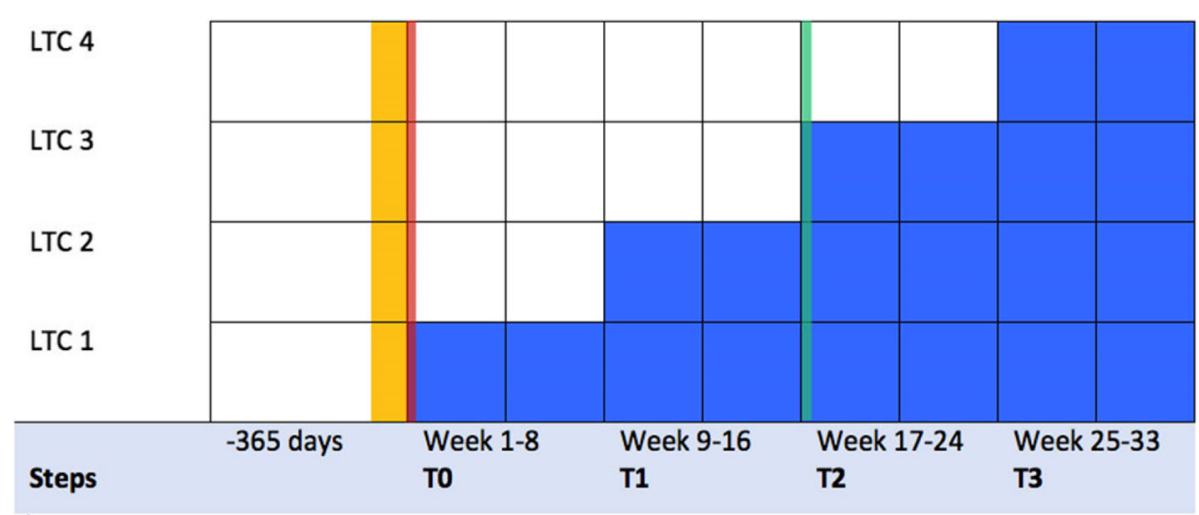

Fig. 1 Schematic of study protocol. Each step from T0 to T3 will be approximately 8 weeks in length. Each cell starting at T0 represents a data collection point. Prior to T0, secondary outcomes will be collected retrospectively collected using the RAI-MDS 2.0. Yellow-shaded cells represent the recruitment period. During this time, LTCs will be invited to participate and will be provided trial information. Consent will be obtained from each LTC. Subsequently, investigator will provide in-person and online information sessions about the trial to potential participants (i.e., clinicians of the participating LTC site), and consent will also be obtained. Randomization (red line) will occur at the beginning of the first step, T0. The GeriMedRisk intervention (dark blue cells) will then be introduced to each LTC in random order. At the beginning of each step, a sealed envelope will be opened to reveal the next LTC to receive the intervention. An interim analysis (green line) will be performed at 16 weeks

GeriMedRisk and telemedicine technology prior to introducing the next site.

\section{Recruitment}

At the commencement of GeriMedRisk access, there will be an initial informative site visit scheduled at a time to reach all the site's LTC clinicians to introduce them to the study and GeriMedRisk including the various methods of accessing the intervention. Investigators will approach the participants (referring clinicians) in meetings facilitated by the long-term care facility at a time that is convenient for the participants. Flyers describing the intervention will be available for posting at the long-term care facility clinical staff areas once the LTC site (cluster) has access to the intervention. Although residents are not subjects of the study, we will notify them or their substitute decision-makers about the study upon the site's introduction to the intervention until its conclusion and provide the option and instructions (LTC site-specific individual and their contact information) to opt out of the service.

\section{Outcomes}

\section{Primary outcome}

Primary outcome measures of this trial are the feasibility and acceptability of the GeriMedRisk intervention, outcome measures, and study design. These include the monthly number and proportion of clinicians who use or decline GeriMedRisk per long-term care home, the number of patients or their substitute decision-makers who decline a GeriMedRisk consultation, and the number of consults, wait times for telephone (ring, queue and call time, or the number of dropped calls), or the
OTN eConsult (defined as the times from the initial consult request to first contact and to when the consultation is provided). We will assess the time required for each consultation and follow-up encounter with the referring clinician. We will quantitatively measure satisfaction by measuring the proportion of GeriMedRisk recommendations that were executed by the referring clinician or the number of repeat referring clinicians. We define a priori threshold for acceptability as:

a) The proportion of GeriMedRisk recommendations that were executed would be $60 \%$ or more $[25,26]$.

b) The proportion of repeat referring clinicians per LTC site would be $>20 \%$ or more.

To identify human resources needs, we will measure the monthly number of consultations requiring physician support with geriatric medicine, clinical pharmacology, and geriatric psychiatry expertise. We will characterize the number of complex cases defined as a case either involving polypharmacy (four or more medications) [27] or requiring physician support or three or more follow-up encounters. We will also assess the financial feasibility of GeriMedRisk through salary and physician billings data [13].

\section{Secondary outcomes}

The core outcome set for effectiveness trials aimed at optimizing prescribing in older adults in care homes informed the selection of our secondary outcomes [28, 29 ]. These outcomes are clinically relevant, potentially modifiable, well-validated in the MDS-RAI 2.0, and in some cases, a surrogate marker of care [30-32]. For 
these same reasons, Health Quality Ontario, a provincial body that advises on the quality of health care provided, also uses these outcomes as long-term care quality indicators [32]. We will assess the completion of our secondary outcome data regarding each LTC site's monthly rates of falls, hospital visits, and medication appropriateness. Using the Resident Assessment Index Minimum Data Set (RAI-MDS) 2.0, we will examine the following in the 1-year lookback period prior to the date of the intervention's introduction and monthly thereafter [30]. We will examine each LTC site's monthly incidence of falls and injurious falls and emergency hospital visits. We will include all-cause mortality and those associated with adverse drug events or falls [28]. We will characterize the proportion of LTC residents receiving potentially inappropriate medications defined by the Beers Criteria and STOPP/START criteria [4, 33], psychotropics (defined as a sedative-hypnotic, antidepressant, antianxiolytic, antipsychotic, lithium or antiseizure medication in the absence of a seizure diagnosis), and long-acting opioids.

\section{Randomization and allocation}

Access to GeriMedRisk (intervention) will be sequentially and randomly rolled out over 8-week periods. The sequence will be randomly generated by a computer model and simple randomization techniques. The allocation sequence will be concealed in sequentially numbered, opaque, sealed envelopes that will be opened at the beginning of each step.

\section{Blinding}

The LTC sites (clusters) will be blinded to the allocation sequence. None of the LTC sites' clinicians or administrators work across the sites. There will also be blinding of the data analysis. The secondary outcomes are systematically collected by nurses, nursing managers, and the LTC administration, in the RAI-MDS 2.0, as part of their mandated reporting to the Ministry of Health and Long-Term Care. By retrospectively collecting the secondary outcomes in the 12 months preceding the intervention (lookback period), this ensures blinded data collection of the LTC patient demographic and clinical variables for this period. During the study, the secondary outcome data will continue to be collected by the same LTC team. Since nurses and nursing managers involved in multidisciplinary patient care are key to geriatric patient care, it would not be ethical to blind them, or any clinicians, involved in the patient's care. The LTC pharmacy records are administrative databases that automatically capture prescription and non-prescription medication use among LTC residents. Only aggregated, anonymized, and de-identified pharmacy records of the LTC will be used for the secondary outcomes. There will be no blinding procedure for patients and their caregivers. We will not remove blinding from individuals involved with data collection or analysis processes. For the qualitative evaluation, there will be no blinding procedure.

\section{Data collection \\ Primary and secondary outcomes}

The primary outcomes of this study include the acceptability and feasibility of GeriMedRisk and the stepped-wedge cluster randomized controlled trial. These include factors influencing the successful implementation of the GeriMedRisk service in terms of feasibility and acceptability among users and non-users and staff providing the GeriMedRisk service. Secondary outcomes include the LTC site's monthly rate of falls, hospital visits, and medication appropriateness. We will measure secondary outcomes in the 52-week period preceding the commencement of GeriMedRisk access (defined as day - 365 to the first day of access to GeriMedRisk) and after, on a monthly basis for each site. These pre-specified outcomes will be systematically collected from our project-specific database from the OTN eConsult database, telephone records, RAI-MDS 2.0, and physician billings.

The Ontario Telemedicine Network (OTN) database is an administrative database that contains physician contact information and specialty and dates of engagement with GeriMedRisk. We will utilize telephone records as measured by the Voice Over Internet Protocol telecommunications manager, Vertical Summit ${ }^{\circ}$, to capture call volumes, dropped calls, and ring, queue, and call times. We will use the RAI-MDS 2.0 data for each LTC site. This standardized assessment tool is routinely applied to all LTC residents in Ontario, Canada, upon LTC admission, readmission from the hospital, change in status, and on a quarterly basis [30-32, 34]. It captures demographical and clinical information that is frequently used in observational research and quality improvement initiatives. We will use de-identified and anonymized aggregate data from the RAI MDS for each LTC site. We will also use aggregated, anonymized, and de-identified LTC pharmacy records to obtain information about prescription and non-prescription medication use.

\section{Qualitative outcomes}

Qualitative data collection will be collected through semi-structured interviews, surveys, and staff consult reflection notes. A team of researchers, not involved in patient care, generated questionnaires based on the theoretical domains framework (TDF) for GeriMedRisk staff, LTC clinicians to understand the barriers, and facilitators to implementation and acceptability of GeriMedRisk [35]. The TDF-based questionnaire will be administered to GeriMedRisk staff throughout the 
intervention implementation. This survey will be sent to all LTC clinicians. We will choose a census sampling approach as the expected numbers of potential GeriMedRisk service users per LTC site will not be large. We will send the questionnaire to a convenience sample of non-referring clinicians, defined as clinicians who practice at the LTC but did not consult during the study period. A total of three email or telephone reminders for the survey will be sent out every 2 weeks to optimize the response rate. Additionally, GeriMedRisk staff will engage in and document self-reflection activities to inform challenges encountered and lessons learned.

\section{Data management}

The OTN, GeriMedRisk clinical database, and pharmacy and electronic medical records are independently managed on secure servers. We will access these databases with encrypted password-protected computers stored in locked cabinets in a secure office. Qualitative study surveys to the referring clinicians, non-referring but eligible clinicians, and GeriMedRisk staff will be administered electronically, data will be de-identified, password-protected, and stored securely. No patient information will be included in the survey. Interviews will be recorded, and data analysis will be stored electronically as a password-protected document on a password-protected encrypted computer in a locked room.

\section{Statistical data analysis Quantitative analysis}

As the main objective of this study is to evaluate the feasibility of GeriMedRisk and its stepped-wedge cluster randomized trial design, we will describe the primary outcomes in a narrative analysis with $95 \%$ confidence intervals (CI) where appropriate. We define success or acceptability of GeriMedRisk as a composite of either $60 \%$ adherence to GeriMedRisk recommendations or at least $20 \%$ of each LTC sites' physicians making more than one consult to GeriMedRisk during the study period (May 1, 2017, to December 31, 2017).

For the secondary outcomes, we will assess each LTC site's outcomes, described as monthly rates, in the 12 months preceding the intervention (baseline) between clusters pre-intervention and calculate the intercluster variance. We will assess for intra- and intercluster differences in the secondary outcomes, described as each LTC site's monthly rates, with weighted generalized linear mixed models. This definition will guide our interpretation of the study's results and potential modifications to the GeriMedRisk intervention or subsequent larger efficacy trial's protocol.

\section{Sample size calculation}

As this is a feasibility study, the primary outcomes include recruitment, acceptability, and time required for the intervention. We used the methods described by Hemming et al. to calculate the sample size for stepped-wedge cluster randomized controlled trials. While the current risk of adverse events at these sites is currently unknown, the emergency department visits rate for LTC in Ontario, Canada, is 25\% [34]. Although LTC clinicians are the users of the intervention, patient outcomes remain of interest, particularly for the larger efficacy trial. In order to detect a decrease in ED visits by $20 \%$, with four steps and one cluster introduced per step and a total sample size of 628 LTC patients, we estimate 181 patients per cluster for $80 \%$ power and $5 \%$ significance [36]. We estimate conservatively that we will need to have consults from $20 \%$ of each LTC's clinicians (physicians, pharmacists, and nurse practitioners). The results of this study will inform our sample size calculation for the future larger efficacy trial.

\section{Qualitative analysis}

For our qualitative analysis, digital recording of the semi-structured interviews will be transformed into verbatim transcripts. Transcripts will be independently reviewed by two members of the research team and analyzed with the purpose of addressing the interview questions. Thematic analysis will be applied to qualitative data from all data collection methods (surveys, interviews, notes) to identify main ideas and themes that cross participant groups (physicians, nurses, pharmacists, GeriMedRisk staff). Quotes that are representative of typical statements given by participants will be extracted to support the themed interpretations. Descriptive data analyses (frequencies: counts, percents, means, SDs, minima, maxima) will be used to describe closed-ended survey data.

We will describe the challenges and accompanying solutions and decision-making process encountered during the study period. These will be informative during our study design process for the future efficacy trial protocol.

\section{Data monitoring and auditing}

Although the GeriMedRisk intervention is in its early stages of development, we will still have a data monitoring committee consisting of JH, JT, SB, and DM. We will conduct an interim analysis after the enrollment of two sites (16 weeks). We will stop the trial if there is significant risk associated with the intervention. While this process will be independent of the trial sponsor (McMaster University), it will not be independent from the investigators due to feasibility.

\section{Harms}

During our follow-up calls, we will actively collect, assess, report, and manage solicited and spontaneously reported adverse events and other unintended effects of 
the trial intervention or trial conduct. These include adverse drug events that occur as a result of GeriMedRisk's suggestions.

\section{Knowledge dissemination}

We plan to disseminate the results of this study in a high-impact peer-reviewed journal within 1 year after the trial end date. We will also share the results with our partners, public and patients, and relevant stakeholders. We will not use professional writers. All authors will fulfill authorship guidelines as defined by the International Committee of Medical Journal Editors. We consulted patients from the community and LTC about GeriMedRisk throughout its conception, study design, and materials (opt-out flyer).

\section{Discussion}

If GeriMedRisk is a feasible intervention, it would be the first technology-based interdisciplinary geriatric pharmacology intervention in Canada. The risks of participating in this GeriMedRisk study include the clinician participant's time involved for each consult and follow-up. Although each consult may take up to $45 \mathrm{~min}$ on the telephone, this time could be significantly decreased by contacting GeriMedRisk through the secure OTN eConsult platform. The benefits of participating in this study include access to geriatric pharmacology expertise in a timely fashion without patient travel to an urban academic health center. Participants will also have the opportunity to provide feedback about GeriMedRisk to help improve the service and its impact on real-world practice. Finally, participants will have access to a service that supports their continuing professional development through concise learning materials pertinent to their patients and quarterly summaries of their learning issues generated from their consults. There will be no cost to participants for these services. By understanding the experiences and perceptions of GeriMedRisk stakeholders, we will be able to provide generalizable information about feasibility and acceptability for the wider health community, refine both referral criteria and mode of delivery for the subsequent clinical trial, inform the potential rollout of the intervention to other healthcare sectors, and further develop the consultation service.

\section{Abbreviations}

ADE: Adverse drug event; Cl: Confidence interval; LTC: Long-term care; OTN: Ontario Telemedicine Network; RAI-MDS: Resident Assessment Index Minimum Data Set

\footnotetext{
Acknowledgements

We thank Ms. Lindsay Cox for the administrative support and Ms. Jane McKinnon-Wilson, Ms. Audrey Devitt, and Ms. Josie D'Avernas for the administrative and organizational support for the GeriMedRisk program. We thank Ms. Susan Brown from the Schlegel Research Institute for Aging for her assistance with the recruitment and enrollment. We thank Ms. Kate Wilson for the administrative support to the GeriMedRisk program and Ms. Anna Tersigni for her expertise in privacy.
}

\section{Funding}

This study is supported by the Labarge Optimal Aging Opportunities Grant 2017-03, Regional Geriatric Program of Hamilton Chair in Aging, Canadian Centre for Aging, and Brain Health Innovation Spark grant. The Canadian Mental Health Association Waterloo Wellington provided in-kind information technology and privacy officer support. The funding bodies had no role in the design of the study and writing of the manuscript nor will they have a role in the collection, analysis, interpretation of data, and manuscript preparation. This study receives no grants from a funding agency in the commercial sector.

\section{Availability of data and materials}

The datasets generated and/or analyzed during the current study are not publicly available due to patient and participant confidentiality but are available from the corresponding author on reasonable request. $\mathrm{JH}, \mathrm{SB}$, and JT will have access to the final trial dataset.

\section{Confidentiality}

Personal information will be stored in clinical databases stored on encrypted password-protected laptops that will be stored in locked cabinets in a locked office. We will have firewall-protected internet connections. OTN, GeriMedRisk, and pharmacy and LTC clinical databases are independently managed on secure connections and servers. We will keep the identities of referring clinicians and their patients confidential. We have consulted with a privacy officer throughout the design of GeriMedRisk and this study protocol.

\section{Authors' contributions \\ $J H, S B, J M, J T, J M P, A H, D M, J A, L T$, and SS contributed to the study concept and design. JH, JT, JA, SB, JMP, AH, LT, JM, and SS contributed to the manuscript preparation. JH, JT, SB, JMP, JA, and SS contributed to the critical review of the manuscript. All authors read and approved the final manuscript.}

\section{Ethics approval and consent to participate}

The Hamilton Integrated Research Ethics Board granted the approval for this study protocol 2017-2812. Following ethics approval, we will start recruiting LTC sites by proactively approaching the LTC site manager and their clinicians (February to May 2017). We will hold scheduled information sessions at each LTC site to describe the intervention and project. The study coordinator will obtain written consent from willing participants (referring clinicians). Participants may also provide verbal and written consent when they call into GeriMedRisk to request a consultation. For participating sites, we will advertise the trial through posters displayed in common clinical staff areas and directly send a flyer to patients and their caregivers which describes the option and procedure to opt out of the intervention. We will track patients who opted out (personally or by caregiver proxy) of GeriMedRisk and inform their clinician of their decision.

\section{Competing interests}

The authors declare that they have no competing interests.

\section{Publisher's Note}

Springer Nature remains neutral with regard to jurisdictional claims in published maps and institutional affiliations.

\section{Author details}

${ }^{1}$ Waterloo Regional Campus, McMaster University DeGroote School of Medicine, 10B Victoria St S, Kitchener, ON, Canada. ${ }^{2}$ Schlegel Research Institute for Aging, 250 Laurelwood Drive, Waterloo, ON, Canada. ${ }^{3}$ Grand River Hospital, 835 King St W, Kitchener, ON, Canada. ${ }^{4}$ St. Joseph's Health Centre Guelph, 100 Westmount Ave, Guelph, ON, Canada. ${ }^{5}$ Department of Family Medicine, McMaster University, 6th floor, 100 Main St W, Hamilton, ON, Canada. ${ }^{6}$ Department of Health Research Methods, Evidence and Impact, McMaster University, H325, 50 Charlton Ave E, Hamilton, ON, Canada. 'Lazaridis School of Business and Economics, Wilfrid Laurier University, 64 University Ave W, Waterloo, ON, Canada. ${ }^{8}$ Ontario Telemedicine Network, 1100-105 Moatfield Drive, Toronto, ON, Canada. ${ }^{9}$ Division of Clinical Pharmacology and Toxicology, McMaster University, 1280 Main St W, Hamilton, ON, Canada. ${ }^{10}$ Li Ka Shing Knowledge Institute, St. Michael's Hospital, 30 Bond St Toronto, Toronto, ON, Canada. ${ }^{11}$ Division of Geriatric Medicine, Department of Medicine, University of Toronto, 190 Elizabeth Street, R. Fraser Elliott Building, 3-805, Toronto, ON, Canada. 
Received: 8 January 2018 Accepted: 21 May 2018

Published online: 20 June 2018

\section{References}

1. Wu C, Bell CM, Wodchis WP. Incidence and economic burden of adverse drug reactions among elderly patients in Ontario emergency departments: a retrospective study. Drug Saf. 2012;35(9):769-81.

2. Canadian Institute for Health Information $(\mathrm{ClHI})$. Adverse drug reaction-related hospitalizations among seniors, 2006 to 2011; 2013. p. 22.

3. Budnitz DS, Lovegrove MC, Shehab N, Richards CL. Emergency hospitalizations for adverse drug events in older Americans. N Engl J Med. 2011;365(21):2002-12.

4. Morgan SG, Hunt J, Rioux J, Proulx J, Weymann D, Tannenbaum C. Frequency and cost of potentially inappropriate prescribing for older adults: a cross-sectional study. C Open. 2016;4(2):E346-51.

5. Page AT, Clifford RM, Potter K, Schwartz D, Etherton-Beer CD. The feasibility and effect of deprescribing in older adults on mortality and health: a systematic review and meta-analysis. Br J Clin Pharmacol. 2016;82(3):583-623.

6. Ailabouni NJ, Nishtala PS, Mangin D, Tordoff JM. General practitioners' insight into deprescribing for the multimorbid older individual: a qualitative study. Int J Clin Pract. 2016;70(3):261-76

7. Ailabouni NJ, Nishtala PS, Mangin D, Tordoff JM. Challenges and enablers of deprescribing: a general practitioner perspective. PLoS One. 2016;11(4):e0151066.

8. Tracy CS, Upshur REG. MedsCheck: an opportunity missed. CMAJ. 2008; 178(4):440

9. Farrell B, Pottie K, Rojas-Fernandez CH, Bjerre LM, Thompson W, Welch V. Methodology for developing deprescribing guidelines: using evidence and GRADE to guide recommendations for deprescribing. PLoS One. 2016;11(8): e0161248

10. Hogan DB, et al. Specialist physicians in geriatrics - report of the Canadian geriatrics society physician resource work group. Can. Geriatr. J. 2012;15(3):68-79.

11. Dorsey ER, Topol EJ. State of telehealth. N Engl J Med. 2016;375(2):154-61.

12. Liddy C, Afkham A, Drosinis P, Joschko J, Keely E. Impact of and satisfaction with a new eConsult service: a mixed methods study of primary care providers. J Am Board Fam Med. 2015;28(3):394-403.

13. Liddy C, Drosinis P, Deri Armstrong C, McKellips F, Afkham A, Keely E. What are the cost savings associated with providing access to specialist care through the Champlain BASE eConsult service? A costing evaluation. BMJ Open. 2016;6(6):e010920.

14. Gray LC, Fatehi F, Martin-Khan M, Peel NM, Smith AC. Telemedicine for specialist geriatric care in small rural hospitals: preliminary data. J Am Geriatr Soc. 2016:64(6):1347-51.

15. Brignell $M$, Wootton $R$, Gray $L$. The application of telemedicine to geriatric medicine. Age Ageing. 2007;36:369-74.

16. Levine SR, Gorman M. 'Telestroke' the application of telemedicine for stroke. Stroke. 1999;30:464:469.

17. I. of M. Committee on poison prevention and control board on health promotion and disease prevention. In: Forging a poison prevention and control system committee. Washington, D.C.: The National Academies Press; 2004.

18. Hubley S, Lynch SB, Schneck C, Thomas M, Shore J. Review of key telepsychiatry outcomes. World J Psychiatry. 2016:6(2):269-82.

19. Arora S, et al. Expanding access to HCV treatment-Extension for Community Healthcare Outcomes (ECHO) project: disruptive innovation in specialty care introduction: the need and significance of a disruptive innovation for specialty care delivery. Hepatology. 2010;52(3):1124-33.

20. Eldridge SM, et al. CONSORT 2010 statement: extension to randomised pilot and feasibility trials. Pilot Feasibility Study. 2016:2(1):64.

21. Waterloo Wellington Local Health Integration Network, Long-term care capacity current state, 2015.

22. Hanlon JT, Schmader KE. The medication appropriateness index at 20: where it started, where it has been and where it may be going. Drugs Aging. 2013;30(11):893-900.

23. Soobiah C, et al. An evaluation of the comparative effectiveness of geriatrician-led comprehensive geriatric assessment for improving patient and healthcare system outcomes for older adults: a protocol for a systematic review and network meta-analysis. Syst Rev. 2017;6(1):65.

24. Dobbins M. Rapid review guidebook steps for conducting a rapid review, Version 1.2. Hamilton: Resource Details | National Collaborating Centre for Methods and Tools; 2017.
25. Aminzadeh F. Adherence to recommendations of community-based comprehensive geriatric assessment programmes. Age Ageing. 2000;29(5):401-7.

26. Georgeton E, Aubert L, Pierrard N, Gaborieau G, Berrut G, de Decker L. General practitioners adherence to recommendations from geriatric assessments made during teleconsultations for the elderly living in nursing homes. Maturitas. 2015:82(2):184-9.

27. Masnoon N, Shakib S, Kalisch-Ellett L, Caughey GE. What is polypharmacy? A systematic review of definitions. BMC Geriatr. 2017;17(1):230.

28. Millar AN, et al. Development of a core outcome set for effectiveness trials aimed at optimising prescribing in older adults in care homes. Trials. 2017; 18(1):175

29. Williamson PR, et al. The COMET handbook: version 1.0. Trials. 2017;18(S3):280

30. Hutchinson AM, et al. The resident assessment instrument-minimum data set 2.0 quality indicators: a systematic review. BMC Health Serv Res. 2010; 10(1):166.

31. Heckman G, Gray L, Hirdes J. Addressing health care needs for frail seniors in Canada: the role of interRAI instruments. Can Geriatr J. 2013:3(1):8-16.

32. Health Quality Ontario, Measuring Up 2017, 2017.

33. Fick DM, Cooper JW, Wade WE, Waller JL, Maclean JR, Beers MH. Updating the beers criteria for potentially inappropriate medication use in older adults. Arch Intern Med. 2003;163(22):2716.

34. Gruneir A, Bell CM, Bronskill SE, Schull M, Anderson GM, Rochon PA. Frequency and pattern of emergency department visits by long-term care residents - a population-based study. J Am Geriatr Soc. 2010;58(3):510-7.

35. Michie S, Johnston M, Abraham C, Lawton R, Parker D, Walker A. Making psychological theory useful for implementing evidence based practice: a consensus approach. Qual Saf Health Care. 2005;14:26-33.

36. Hemming K, Haines TP, Chilton PJ, Girling AJ, Lilford RJ. The stepped wedge cluster randomised trial: rationale, design, analysis, and reporting. BMJ. 2015; 350(1):h391.

\section{Ready to submit your research? Choose BMC and benefit from:}

- fast, convenient online submission

- thorough peer review by experienced researchers in your field

- rapid publication on acceptance

- support for research data, including large and complex data types

- gold Open Access which fosters wider collaboration and increased citations

- maximum visibility for your research: over $100 \mathrm{M}$ website views per year

At BMC, research is always in progress.

Learn more biomedcentral.com/submissions 